\title{
I mirabili discorsi di Monsieur Palissy
}

\author{
Francesco Luzzini \\ Università degli Studi di Milano - Dipartimento di Bioscienze \\ francesco_luzzini@yahoo.com
}

Theorique - «Mi credete tanto ignorante da fidarmi più delle vostre parole che del parere d'un gran numero di filosofi, per cui le acque provengono dal mare e vi fanno ritorno? Persino le vedovelle anziane la pensano così, e noi con loro. Siete molto presuntuoso a volerci proporre una dottrina del tutto nuova, quasi foste il più abile dei filosofi».

Practique - «Sono sicuro di poter vincere contro di voi, e contro tutti quelli della vostra opinione. Persino contro Aristotele, e con lui i più eccellenti filosofi d'ogni tempo: giacché son certo che la mia idea è corretta. [...] Se l'origine delle fonti fosse nel mare, come potrebbero queste prosciugarsi d'estate, quando il mare non è più basso che in inverno? [... . Vi do per regola certa e generale, che le acque non s'alzano mai più in alto delle sorgenti da cui provengono. Non sapete forse esserci più fontane nei monti che in valle: e che se anche gli oceani fossero alti quanto le più alte montagne, sarebbe comunque impossibile per le sorgenti venire dal mare? La ragione si è, che facendo scendere l'acqua da una certa altezza, e volendo farla risalire fino a un'altezza pari a quella di prima, questa dovrà passare in un tubo ben chiuso, così da evitare perdite: altrimenti l'acqua non salirà, ma scenderà ancora per la prima apertura disponibile» (Palissy 1580).

Theorique e Practique sono due personaggi evidentemente contrapposti; il primo personificazione (ottusa e caricaturale) delle vecchie dottrine, l'altro dell'esperienza diretta, staccata da qualsiasi interpretazione filosofica. L'anno è il 1580, e lo schema retorico utilizzato - quello del dialogo polemico - è un classico della trattatistica dell'epoca. Lo rimarrà ancora per un bel pezzo: il suo caso più emblematico vedrà la luce mezzo secolo dopo, con la pubblicazione del Dialogo sopra $i$ due massimi sistemi del mondo (Galilei 1632).

Come nella celebre opera galileiana, anche qui la parzialità dell'autore è evidente. In questo caso, anzi, le cautele dissimulatorie sembrano ancora più blande. E studiando l'estrazione professionale, la vita e le opere di questo autore, il ceramista e idraulico francese Bernard Palissy (1510-1589), riesce ancora più semplice intuirne il pensiero. Il suo alter ego è Practique: e l'opera, intitolata Discours admirables de la nature des eaux et fonteines, rappresenta una vera e propria dichiarazione di guerra contro qualsiasi speculazione che non si fondi su basi empiriche.

Proprio la continua insistenza sulla superiorità della ricerca pratica sulla filosofia distanzia Palissy dagli autori coevi o precedenti, in un'epoca in cui gli studi della natura che non fanno riferimento (anche solo formale) al pensiero dei maestri classici, Aristotele in primis, sono ancora visti con grande sospetto. I Discours admirables non si limitano a ignorare i filoso- fi, ma li contestano apertamente; e osano affrontare un tema che, per la sua importanza e difficoltà, nel XVI secolo ha già all'attivo attenzioni illustri e feroci dibattiti. L'enigma è lo stesso che intrigherà generazioni di studiosi nei due secoli a venire: qual è l'origine delle acque dolci? Le piogge, le nevi, i ghiacciai sono sufficienti a ricaricare le sorgenti e le falde? O forse esistono altri, occulti fenomeni in grado di privare del sale l'acqua marina e di trasportarla sui monti?.

Ancor prima di Palissy, queste domande avevano impegnato sapienti e pratici di varia estrazione culturale, religiosa e professionale, spesso dediti a eclettici interessi: dal mineralogista, medico e filologo tedesco Georg Agricola (1494-1555) al matematico, meccanico e pastore evangelico Jacques Besson (1540-1573); dal filosofo Girolamo Cardano (1501-1576), che si occupò anche di medicina, meccanica, matematica e astrologia, a Leonardo da Vinci (1452-1519), considerato ancora oggi la mente poliedrica per antonomasia. Nessuno di costoro, tuttavia, rinunciò a integrare i propri dati sperimentali (spesso frutto di solidissime esperienze sul campo) in un sistema interpretativo filosoficamente ben definito. Lo stesso Agricola, che dell'attività mineraria e delle scienze della Terra fu il più competente studioso del suo periodo, si affidò in più casi all'autorità degli antichi. E nel De ortu et causis subterraneorum (Agricola 1546), discutendo il fenomeno della misteriosa ricarica delle sorgenti, non poté escludere che canali sotterranei congiungessero i mari alla terraferma, consentendo il passaggio e la desalinazione dell'acqua; o che l'aria potesse condensare e dare origine a grandi quantità d'acqua dolce, raffreddandosi nelle cavità dei monti. Fenomeni, questi, ritenuti indispensabili per le regioni aride («quibus regionibus imbres a natura fere sunt denegati»).

Nei Discours admirables non c'è spazio per le teorie classiche delle vene sotterranee e della filtrazione attraverso gli strati rocciosi, che Palissy - per bocca di Practique - critica aspramente. Non a caso l'autore è un semplice artigiano, non un letterato. Le sue idee sono legate a filo doppio alla sua pluridecennale esperienza come ceramista (quando pubblica il trattato ha ormai settant'anni), che gli ha confermato l'impossibilità per la terra e le rocce di trasformare l'acqua salata in dolce. Non c'è bisogno, d'altronde, di ricorrere a meccanismi tanto occulti: l'acqua caduta con le piogge invernali è sollevata in vapore dal sole estivo e dai venti asciutti, e di nuovo torna al mare l'inverno seguente («les eaux des pluyes qui tombent en hyuer remontent en esté, pour retourner encores en hyuer»). È quindi dalle nubi, che vanno in ogni dove come «araldi inviati da Dio» («comme heraux enuoyez de Dieu»), che proviene l'acqua in grado di ricaricare falde e sorgenti. Quanto al cosid- 
detto sistema dei lambicchi, per cui buona parte delle fonti ha origine per la condensazione di un'«aria spessa» nelle profondità delle caverne, non lo si può negare completamente. Non è forse vero, del resto, che il vapore in una pentola sul fuoco si trasforma in acqua sotto il coperchio? Ma di certo questa non è la sola causa; giacché fin dalla creazione del mondo le fontane, i fiumi e i ruscelli hanno continuato a sgorgare e fluire dai monti, e sarebbe impossibile per le sole caverne fornire abbastanza acqua per un anno, o anche solo per un mese («il est impossible que les dittes cavernes peussent fournir d'eau pour une annee non pas pour un mois, autant de fluves qui d'escoulent iournellement») (Palissy 1580).

L'intransigenza di Palissy, per sua disgrazia, non si limitò alla (comunque pericolosa) battaglia contro i filosofi. Con altrettanta ostinazione difese la sua fede ugonotta, proprio quando la Francia si stava preparando a voltarle bruscamente le spalle. Già nel 1572, durante l'orrenda notte di S. Bartolomeo, era scampato per un soffio alla strage. Ma non sfuggì all'arresto nel 1588, tre anni dopo la revoca degli editti di tolleranza religiosa imposta dal Trattato di Nemours. Avendo rifiutato di convertirsi, venne condannato a morte e rinchiuso della Bastiglia. Ma l'età veneranda e le terribili condizioni della prigionia gli risparmiarono il boia, facendolo morire di stenti nel giro di un solo anno.

È stato evidenziato come le ragioni esposte nei Discours admirables siano «sensate ma non certo nuove» (Maffioli 2010). L'intuizione della ricarica delle sorgenti per mezzo delle sole acque meteoriche, infatti, era già ampiamente diffusa nel XVI secolo, e non di rado interagì e s'integrò con le teorie dei passaggi sotterranei e dei lambicchi. Besson, ad esempio, aveva espresso idee simili nel suo trattato, Lart et science de trouvers les eaux et fontaines (Besson 1569). Questa constatazione, tuttavia, non sminuisce il valore dell'opera di Palissy, che nella sua feroce polemica contro i filosofi esprime istanze che lo accomunano più ai dibattiti del XVII secolo che al panorama culturale cinquecentesco. E a costo di scadere in un insidioso (ma, si spera, perdonabile) precursionismo, è difficile resistere alla tentazione di notare le affinità di Theorique e Practique con Simplicio e Salviati: i protagonisti di quel più celebre Dialogo che, cinquant'anni dopo, avrebbe ufficializzato le rivendicazioni dello sperimentalismo contro il principio d'autorità, inaugurando uno dei più fertili, complessi e affascinanti periodi della storia della scienza.

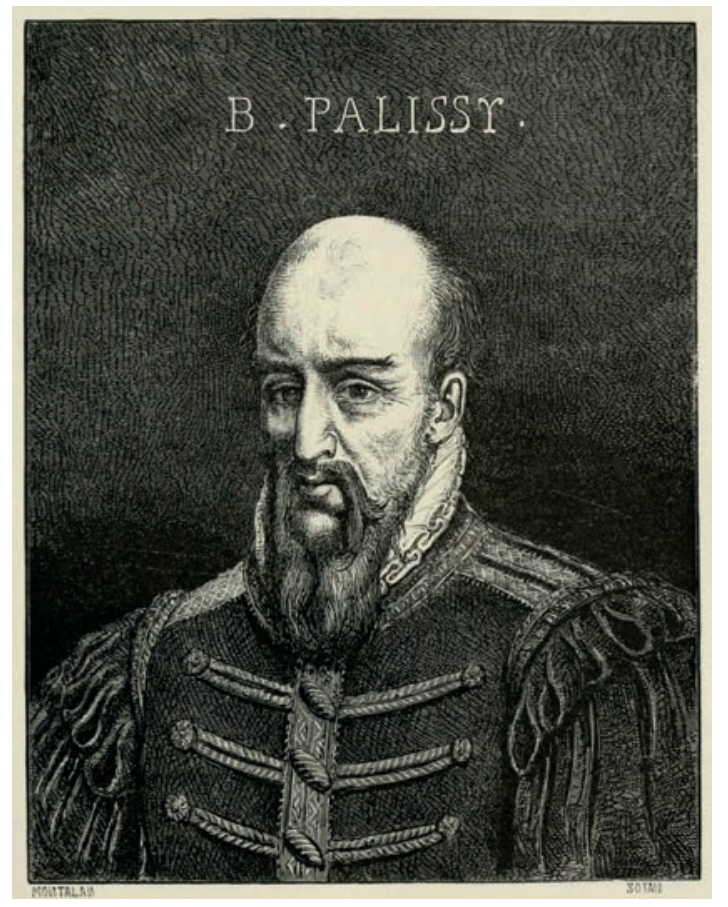

Fig. 1 - Bernard Palissy - ritratto.

\section{BIBLIOGRAFIA}

Agricola (Bauer G., 1546) - De ortu et causis subterraneorum lib. V. Per Hieronimymum Frobenium et Nic. Episcopum, Basileae, pp. 3-12.

Besson J. (1569) - L'art et science de trouvers les eaux et fontaines cachees soubs terre, autrement que par les moyens vulgaires des agriculteurs et architectes. Pour P. Trepperel, Orleans.

Galilei G. (1632) - Dialogo sopra i due massimi sistemi del mondo, tolemaico, e copernicano. Per Giovanni Battista Landini, Firenze.

Maffioli C.S. (2010) - La via delle acque (1500-1700). Appropriazione delle arti e trasformazione delle matematiche. Olschki, Firenze, pp. 11, 101-102, 124-125, 146, 163.

Palissy B. (1580) - Discours admirables de la nature des eaux et fonteines, tant naturelles qu'artificielles..., Paris, Chez M. le Ieune, a l'enseigne du serpent, devant le college de Cambray, Paris, pp. 34 35, 40-45. Le traduzioni italiane sono opera mia. 